\title{
Interactive comment on "Study on variations in lidar ratios for Shanghai based on Raman lidar" by Tongqiang Liu et al.
}

\section{Tongqiang Liu et al.}

oxeye75@163.com

Received and published: 16 February 2021

Anonymous Referee \#1 Interactive comment on "Study on variations in lidar ratios for Shanghai based on Raman lidar" by Tongqiang Liu et al.

The aerosol types are very important information in assessing the impacts of aerosols on climate forcing. Accurate measurements of Lidar ratios (LR) and better understanding of their variation characteristics provide a way from remote sensing for the scientific research on aerosol type information. This study addresses the results of a long-term observation of LR at $355 \mathrm{~nm}$ of Raman lidar in Shanghai from 2017 to 2019, and analyzed their variations and influencing factors. This kind of observations about LR are rare and worth encouraging, especially over Eastern Asia region. I recommend a minor 
revision before publication with ACP. The detail comments or suggestions are shown below.

Reply: We are very grateful for the referee's encouraging comments and recommendations for publication with minor revision. The following are our point-by-point responses to the referee's comments.

My general concern about this study is that although the data results are rare and good, Some of the analysis on the reasons for LR vertical and temporal variations and aerosol source influences are not straightforward. Several inferences and methods in part 3 seems too complicated and sometimes confused. First, it is difficult to conclude aerosol type according to the comparison of LR data with some literature reports in table 1 of the manuscript. Second, the results from the usage of cluster analysis of back trajectories in 3.2.2 are not significant without a statistical test. In fact, the back trajectory analysis should be effective for long-range transport dominated aerosols. Over an urban area of Shanghai, I suppose the status of aerosols from long-time averaged perhaps are mainly dominated by local urban emissions. Dust fraction in the aerosols should be a main factor to affect the LR data and volume depolarization ratios $(\delta)$, sometimes absorbing aerosols from primary aerosols may also cause an increase of LR in the surface layer. I suggest a study to check the dust fraction on LR and $\delta$ only with the surface PM2.5/PM10 data. The PM2.5/PM10 data should be easy to obtain over urban area of Shanghai. If the authors have more chemical composition observations, for examples, EC, that will be better.

Reply: We thank the referee for providing insightful comments and constructive suggestions, which have been very helpful for us to improve the manuscript. The identification of aerosol type can not only use LR, but also needs other physical parameters such as depolarization ratio and color ratio. Therefore, the analysis on aerosol type identified by LR in section 3.1.1 is not accurate and we've deleted them. We conducted significance tests on cluster analysis results in section 3.2.2 according to referee's suggestions, and the difference of LR $(\delta)$ between the four clusters was significant. In

Interactive

comment
Printer-friendly version

Discussion paper 
addition, the backward trajectory analysis in Section 3.2.2 was mainly to explore the impact of aerosol sources on LR, so we set the airflow reaching altitude at $1000 \mathrm{~m}$ above sea level. Generally, aerosols above PBL are mainly affected by long-range transport. Fig. 1 shows the mean value of PM mass concentration of four clusters. The PM mass concentration data obtained from observations near the Raman lidar site. Lower PM2.5/PM10 indicates significant contributions by coarse model aerosols such as dust, and higher PM2.5/PM10 is attributed to anthropogenic aerosols such as sulfate and nitrate (Tian et al., 2018). The mean PM2.5/PM10 affected by the aerosols brought by air mass 4 is 0.63 , lowest in the four clusters, which indicated that coarse mode particles contribution is significant. This is different from the result in 3.2.2 that the contribution of spherical smoke particles brought by air mass 4 is larger. The discrepancy may be due to the fact that PM data come from ground sampling, which is difficult to evaluate the impact of long-range transport aerosols above PBL on LR.

1. the English of the paper should be improved, for examples, some definite articles 'the' be misused.

Reply: We appreciated the referee's suggestions. We reviewed the manuscript carefully and have tried our best to corrected grammatical errors and improve the English of the paper.

2. Line 43 , "The $P(\pi)$ was related to sphericity of the particle which can be obtained from the polarization lidar", can it be obtained from the polarization lidar?

Reply: We appreciated the referee's suggestions. The phase function at $180^{\circ}(\mathrm{P}(\pi))$ can't be obtained from polarization lidar, and we have revised it. $\mathrm{P}(\pi)$ was related to sphericity of particles, and the sphericity information of particles can be obtained from polarization lidar.

3. Line 46, "Moreover, the heating effect of the absorbing aerosol on the atmosphere results in an increase of atmospheric stability and a reduction of atmospheric vertical exchange, which further aggravates the accumulation of pollutants (absorbing parti-

Printer-friendly version

Discussion paper
Interactive

comment 
cles) and a positive feedback is established". Whether this conclusion is right, I think it is depending on a suitable vertical distribution of the absorbing aerosol. If the absorbing aerosols are on near surface layer their heating effect will enhance the instability of the atmospheric boundary layer.

Reply: We agree with referee's concerns and have revised it.

Moreover, absorbing aerosols increases atmospheric stability by reducing the solar radiation reaching the surface during the day. On the contrary, absorbing aerosols on near surface layer heats the surface and increases the atmospheric instability during night (Jacobson, 1998; Jacobson and Kaufman, 2006).

4. Line 92, "the Raman signals are very weak in the daytime", are the Raman signals stronger in the night time than in the daytime, I think they are same, but signal-to-noise ratios are different at daytime and nighttime.

Reply: We agree with referee's concerns. The intensity of Raman signals is the same in daytime and night time, but signal-to-noise ratios of Raman signals in daytime are much lower than that in night time. We've revised this sentence.

Since Raman Lidar used in this study can detect the Raman scattering signal of $387 \mathrm{~nm}$ nitrogen and signal-to-noise ratios of Raman signals in daytime are much lower than that in night time, the $355 \mathrm{~nm}$ LR at night can be obtained through the retrieval.

5. Line 111-115, and 338-340, $\mathrm{RH}$ data from a model simulation or reanalysis data are not credible. I do not think this analysis is useful to the study.

Reply: We appreciate the referee's suggestions and add some discussion about the reasons of using $\mathrm{RH}$ data from ERA5.

In recent years, some studies have evaluated the accuracy of reanalysis data provided by the European Center for Medium-Range Weather Forecasts (ECMWF) based on radiosonde data. For example, Luo et al. (2020) found that the average $\mathrm{RH}$ discrepancy between ERA-Interim radiosonde was within $10 \%$ below $500 \mathrm{hPa}$. Song et al. (2020) 
found that the root mean square error (RMSE) of ERA5 RH was 3.85\% compared with the $\mathrm{RH}$ profile of the radiosonde. The above results show that $\mathrm{RH}$ from reanalysis data has good accuracy, and has been widely used in various research fields (Sajadi et al., 2020; Tzanis et al., 2019; Xiao et al., 2020).

In addition, we compared the water vapor mixing ratio of Raman lidar with that of ERA5. As shown in Fig. 2, the water vapor obtained from Raman lidar has good consistency with that of ERA5, and the correlation coefficient is 0.94 . The comparison between Raman lidar retrieved results and reanalysis data will be put into our next paper.

6. In 3.1.1 General variation of LR. Some comparisons with those values in table 1, then conclude the aerosol type, I do not agree with this method. Dust fraction, or fine-mode fraction, maybe is more direct to help the analysis of the aerosol type.

Reply: The referee's comments are very valuable. Dust fraction or fine-mode fraction combined with LR really helps to analyze aerosol types more reasonably. The finemode fraction can be determined indirectly by the ratio of extinction coefficients of two wavelengths (color ratio) (Liu et al., 2017). Our Raman lidar can only obtain accurate extinction coefficient profile at $355 \mathrm{~nm}$, but cannot obtain accurate extinction coefficient profile at $532 \mathrm{~nm}$ due to lack of the corresponding Raman channel. Therefore, the accurate color ratio cannot be obtained, which leads to the inaccurate determination of aerosol particle size. According to definition of LR in Müller's (Müller, 2003) study, LR depends on the absorption properties and phase function of the aerosol. In fact, aerosol absorption properties and phase function can't be obtained only from Raman lidar observation at present. Therefore, we just tried to analyze possible aerosol types by comparing our LR values with those of typical aerosols in previous studies. We agree with your suggestions and have deleted the corresponding analysis on aerosol type identified by LR in section 3.1.1. In order to more accurately analyze the impact of changes in aerosol types on LR, we will use collaborative observation of drones to obtain vertical variation of aerosol absorption properties and size distribution in the future.

Printer-friendly version

Discussion paper
Interactive

comment

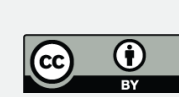


7. Line 172, "Above $2 \mathrm{~km}$, the mean values of $\mathrm{LR}$ were usually less than $40 \mathrm{sr}$, which alluded to an increasing influence of background aerosol (i.e. less absorbing coarsemode particle)", I think "coarse-mode particle" is not accurate. The coarse-mode particle will lead an increase of LR. Fine-mode secondary aerosols are dominated in longrange transport process over an urban area.

Reply: We appreciate the referee's suggestions. Ferrare et al. (2001) pointed out that $\mathrm{LR}$ at $355 \mathrm{~nm}$ increased with the increase of the relative amount of fine model aerosols. The small LR at high altitude were usually related to low aerosol concentration and low absorption efficiency of aerosols (Hänel et al., 2012; Hee et al., 2016). Therefore, we've revised the sentence.

Above $2 \mathrm{~km}$, mean values of LR were usually less than $40 \mathrm{sr}$, which was related to low aerosol concentration and low absorption efficiency of aerosols (Hänel et al., 2012; Hee et al., 2016).

8. Results and analysis related to Figure 4, I do not think the current observation period of the data support a seasonal or monthly/annual change analysis. The observation period presented by Fig. 1 only include some individual months over the 3 year respectively, it is not reasonable to combine the individual months from different years to an annual or seasonal change. The authors need longer and more continuous data.

Reply: We agree with referee's comments that it is more reasonable to use longer and more continuous data to analyze the seasonal or monthly variation of LR. However, it can be seen from Table R1 and R2 that although Raman lidar observation time is only three years, it covers all months, especially in 2018, which has been continuously observed for almost the whole year. In addition, there are enough samples in different months/seasons, and the average LR have higher statistical power. Therefore, we believe that monthly/seasonal variations of LR are still credible, and the LR monthly/seasonal variations also provides reliable observation basis for the correction of parameters from elastic lidar. Annual variation of LR wasn't analyzed due to the 
short observation time. Table 1 Number of effective observation hours in different season during Raman lidar observation period. Season Spring Summer Autumn Winter Number 27221657122 Table 2 Number of effective observation hours in different month during Raman lidar observation period. Month Jan Feb Mar Apr May Jun Jul Aug Sept Oct Vov Dec Number 38357511582961002022152049

9. Figure 5, I suggest date labels for the $x$-axis instead of sequence numbers.

Reply: The $\mathrm{x}$-axis of Figure 5 has been marked by date (Day/Month/Year) according to referee's suggestions.

10. Analysis related to Figure 7 with back trajectory cluster analysis, I do not think it is significant.

Reply: We agree with referee's concerns and have added significance tests of the results from cluster analysis in the revised manuscript.

Backward trajectory cluster analysis based on HYSPLIT model is widely used in atmospheric aerosol research (Wang et al., 2020; Xu et al., 2018; Zhang et al., 2020). We performed a significance test on the results of the cluster analysis, and the one-way ANOVA showed that $P<0.05$, indicating that the LR of the four clusters were significantly different. Similarly, there were significant differences in $\delta$ among the four clusters.

In addition, we have added the percentage of each cluster in Fig 7 (a).

11. Analysis related to Figure $8 \& 9$, why not to check by some surface observations instead of only using AOD, for examples, PM2.5 mass concentration?

Reply: The referee's comments are very valuable. Fig. 3 shows the vertical variation of LR at different PM2.5 mass concentrations. The slope of LR vertical variation didn't show a decreasing trend with the increase of PM2.5 mass concentration. In addition, Fig. 4 shows the hourly variation of PM2.5 in five cases with abnormal LR variation. Except for case 2, the PM2.5 mass concentrations in the other four cases

Printer-friendly version

Discussion paper 
were larger. PM2.5 data can well reflect surface pollution degree of particulate matter, but it is difficult to identify aerosol types, especially above $1 \mathrm{~km}$ altitude. Therefore, spatial distribution of tracer were used to determine whether these five cases were affected by biomass burning aerosols.

Interactive comment on Atmos. Chem. Phys. Discuss., https://doi.org/10.5194/acp-2020-1162, 2020.

comment 
ACPD

Interactive

comment

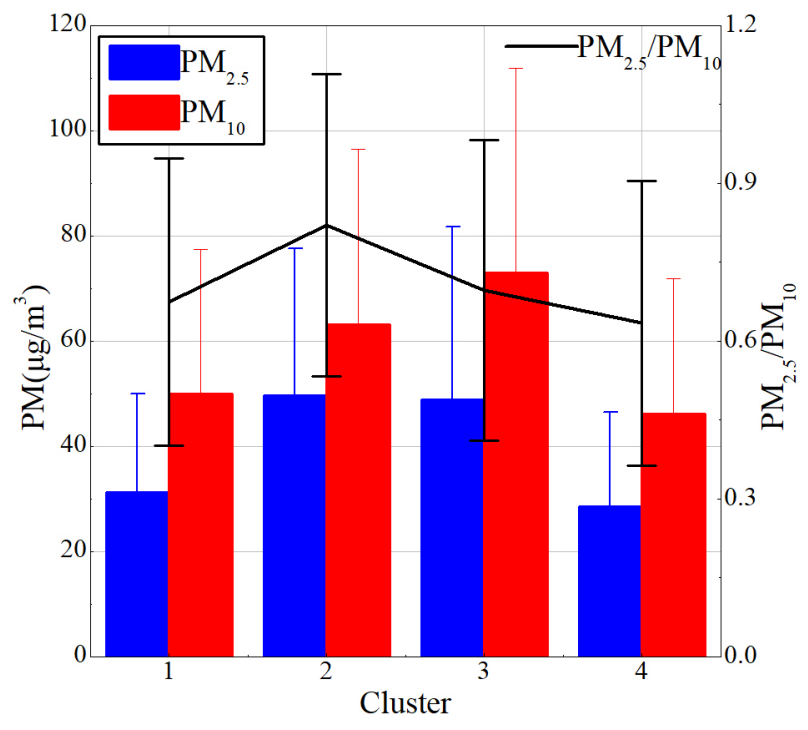

Fig. 1. PM of four clusters.

Printer-friendly version

Discussion paper 
Interactive

comment

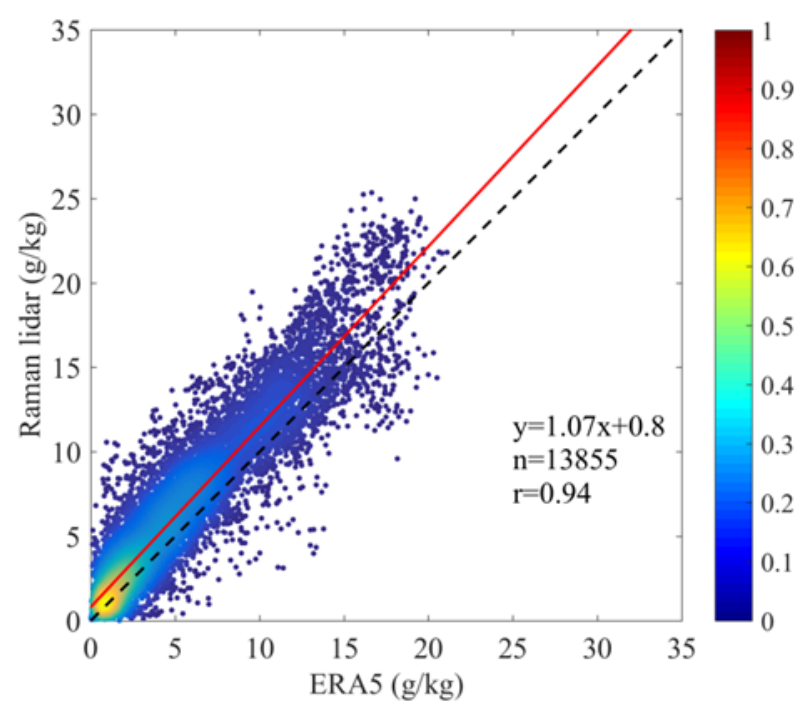

Fig. 2. Comparison of water vapor mixing ratio between Raman lidar and ERA5. 


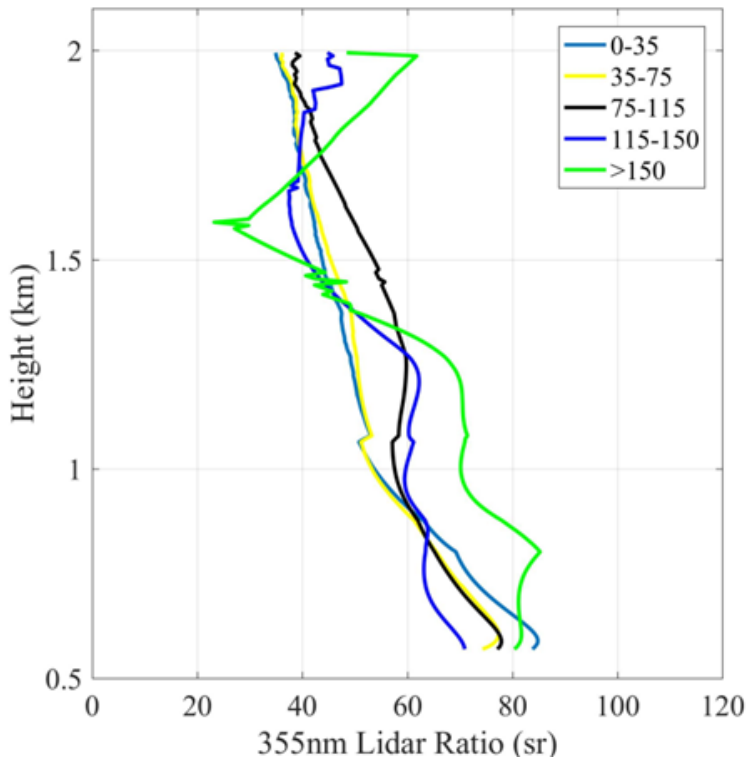

Interactive

comment

Fig. 3. LR profiles in different PM2.5 mass concentration intervals. 


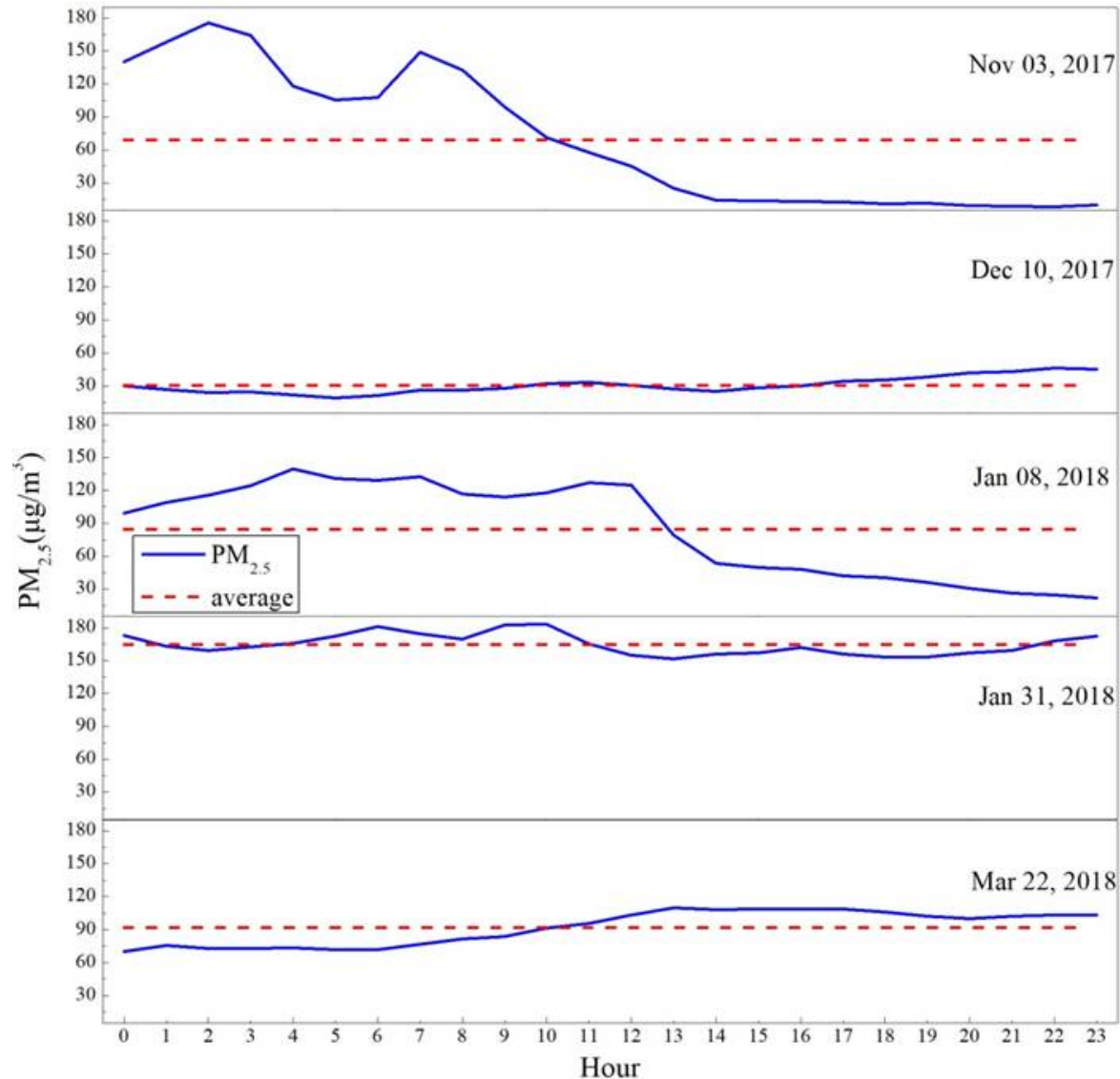

Fig. 4. The hourly variation of PM2.5 mass concentration in five cases, and red dotted line is daily average value.
ACPD

Interactive

comment 